\title{
NIVEL DE APROPIACIÓN DE LA IDEA \\ DE DISCONTINUIDAD DE LA MATERIA \\ EN ALUMNOS DE BACHILLERATO. IMPLICACIONES DIDÁCTICAS
}

GENTIL GONZÁLEZ, C. (1). IGLESIAS BLANCO, A. (2), OLIVA MARTÍNEZ, J.M.(3)

(1) Centro de Profesores de Cádiz

(2) I.B. Isla de León. San Fernando. Cádiz

(3) Wenceslao Benítex. San Fernando. Cádiz

\section{SUMMARY}

In this paper we will try show how those pupils starting Secondary Education have scarcely assumed the discontinuous nature of matter and little progress about it is noticed at the end of their studies.

\section{INTRODUCCIÓN}

Diversos autores (Martinand 1986, Rosado 1986) han resaltado ya la importancia inherente a los procesos de aprendizaje que implican el uso de modelos cientificos por parte de los alumnos. Concretamente, en el campo particular de la Química adquiere especial relevancia el estudio de la estructura interna de la materia y de la naturaleza de las reacciones químicas mediante la utilización de un modelo atómico. Bajo un enfoque constructivista, la ensentanza se concibe como un proceso de reestructuración de los esquemas conceptuales previos (Driver 1986), y es por ello por lo que resulta de sumo interés conocer las ideas que poseen los alum. nos que comienzan la Química en el bachillerato (15-17 años) acerca de la estructura interna de la materia con el fin de adoptar estrategias concretas en clase que posibiliten un aprendizaje significativo del carácter discontinuo de la materia.

La investigación realizada, tanto dentro (Enciso, Llorens y Sendra 1987, Furió y Hernández 1983, Llorens 1987) como fuera de nuestras fronteras (Novick y Nussbaum 1978, 1981; Mitchell y Kellington 1982; Driver 1983; Brook et al. 1984; Anderson 1984) con alumnos de estas edades parece haberse centrado, fundamentalmente, en la detección de las ideas alternativas que usan los estudiantes cuando son instados a interpretar fenómenos físicos y químicos en términos de un modelo corpuscular. Este tipo de ideas parece girar en torno a una escasa noción de la existencia de vacio entre par. tículas, un rechazo a la idea de interacción entre las mismas, una imagen molecular estática y una tendencia a transmitir a las partículas las propiedades y atributos del mundo macroscópico, dando lugar a explicaciones tales como que las particulas mismas cambian de estado, se dilatan, etc.

Como resultado de estos estudios, se ha podido poner de manifiesto que, en general, los adolescentes tienen una imagen de la materia como si de algo continuo se tratase, lo cual no debiera extrañarnos si tenemos en clienta que incluso históricamente la ruptura definitiva con la visión aristotélica de la materia no se produjo hasta épocas bastante recientes.

Mediante la presente investigación se pretende comprobar hasta qué punto los alumnos que comienzan el bachillerato en nuestro pais tienen asumida la dișcontinuidad de la materia, y en qué medida la enseñanza recibida durante $2^{\circ}, 3^{\circ}$ de BUP y COU contribuye a mejorar esta situación. En forma operativa estos objetivos se pueden concretar a través de los siguientes interrogantes:

- Hasta qué punto los alumnos utilizan espontáneamente un modelo corpuscular a la hora de interpretar fenómenos físicos cotidianos?

- ¿Con qué consistencia emplean los alumnos un modelo corpuscular a la hora de interpretar o predecir diversos fenómenos similares?

- Hasta qué punto los alumnos tienden a utilizar un modelo corpuscular a la hora de predecir el resultado 
de un fenómeno como es la conservación de la masa en un proceso físico?

- Qué diferencias pueden observarse entre los alumnos que comienzan y finalizan la enseñanza media?

\section{DISEÑO EXPERIMENTAL}

El cuestionario empleado (ver anexo) constaba de 12 items de respuesta abierta, una de las cuales fue desglosada en dos subapartados (6A y $6 \mathrm{~B})$. A través del mismo, los alumnos fueron instados a realizar interpretaciones o predicciones, según el caso, acerca de diversos fenómenos físicos, algunos de ellos íntimamente relacionados con las experiencias cotidianas. En ningún caso se orientó a los chicos acerca del modelo que deberían utilizar en sus explicaciones.

El cuestionario se administró a principios de Febrero del curso 87-88 a una muestra formada por 154 alumnos/as (105 de $2^{\circ}$ de BUP y 49 de COU), procedentes de los dos institutos públicos de San Fernando (Cádiz). Los alumnos de $2^{\circ}$ no habian recibido aún enseñanza de Química durante el bachillerato, mientras que los de COU habian estudiado ya algunos de los primeros temas correspondientes al programa oficial de dicho curso.

Las respuestas dadas por los chicos se analizaron bajo dos puntos de vistas. Por un lado, se clasificaron según los tipos de respuestas y explicaciones empleadas, y por otro, se contabilizó el número total de explicaciones realizadas a nivel microscopico, considerando como tales todas aquéllas que incluian de forma explícita términos tales como partículas, moléculas, átomo, ión, enlace, etc.

La validez de los ítems, o capacidad para discernir entre alumnos atomistas y no atomistas, se midió a través del indice de validez o discriminación Id (Sampascual 1982), considerando la muestra de una forma global $(\mathrm{N}=154)$.

Para calcular correlaciones entre ítems se determinó el coeficiente de correlación phi $(\varnothing)$, equivalente al $r$ producto-momento de Pearson, pero adaptado a casos en los que los datos estadísticos presentan dicotomias (Garrett 1983). Dichos coeficientes se obtuvieron a partir de las tablas de contingencia correspondientes.

\section{RESULTADOS Y DISCUSIÓN}

\subsection{Utilización explícita de la idea de discontinuidad}

Ła Tabla I recoge los porcentajes de alumnos que utilizaban abierta y explícitamente un modelo corpuscular durante las explicaciones empleadas en cada una de las cuestiones. No se ha tenido en cuenta en los cálculos si las explicaciones eran correctas o no, ya que es- tos valores trataban únicamente de determinar la importancia que los chicos atribuían a la utilización expresa de la idea de discontinuidad a la hora de interpretar un fenómeno físico, lo cual se ha considerado como una medida indirecta del grado de apropiación de dicha idea.

Se observa, en general, una escasa tendencia en los alumnos de $2^{\circ}$ a utilizar explicaciones de tipo micros. cópico, si bien se aprecian notables diferencias de unos casos a otros según el contenido de cada cuestion. En muchos casos, incluso, los chicos daban interpretaciones atomistas que diferían notablemente de aquéllas que pueden considerarse como aceptables dentro del con. texto escolar.

Cuando se analizan los resultados correspondientes a los individuos de COU se aprecia una escasa mejora, constatándose diferencias significativas tan sólo en tres de las cuestiones propuestas (cuestión $5 \mathrm{p}<0.01$, cuestiones 7 y $11 \mathrm{p}<0.001$ ).

A continuación, se hace especia! referencia a las cuestiones $1,5,6 \mathrm{~A}, 8,9, \mathrm{y} 11$, ya que es en estos casos donde, como puede apreciarse (Tabla I), los índices de discriminación alcanzan valores más altos.

Tabla I

$\%$ Explicaciones atomistas.

\begin{tabular}{|c|c|c|c|}
\hline CUEGTION NO & $\begin{array}{c}29 \text { Btyp } \\
(N=105)\end{array}$ & $\begin{array}{c}\text { COU } \\
(N=49)\end{array}$ & Id \\
\hline $\begin{array}{cc}- & 1 \\
& 2 \\
& 3 \\
& 4 \\
-\quad & 5 \\
-\quad 6 A \\
& 69 \\
& 7 \\
-\quad 8 \\
-\quad 9 \\
-10 \\
-\quad 11 \\
12\end{array}$ & $\begin{array}{r}26 \\
15 \\
5 \\
15 \\
16 \\
52 \\
15 \\
22 \\
22 \\
34 \\
9 \\
35 \\
17\end{array}$ & $\begin{array}{l}32 \\
26 \\
16 \\
18 \\
46 \\
69 \\
28 \\
65 \\
36 \\
44 \\
16 \\
73 \\
34\end{array}$ & $\begin{array}{l}0.55 \\
0.33 \\
0.14 \\
0.23 \\
0.51 \\
0.59 \\
0.39 \\
0.43 \\
0.53 . \\
0.57 \\
0.27 \\
0.67 \\
0.47\end{array}$ \\
\hline
\end{tabular}

\subsection{Consistencia en el uso de la idea de discontinuidad}

La Tabla II nos muestra los coeficientes de correlación $(\varnothing)$ inter-items calculados para diversas parejas de cuestiones. Estos valores se han utilizado como una medida de la consistencia en el uso del modelo a través de diferentes contextos, de forma similar a como ya lo han hecho otros autores (Engel Clough y Driver 1986) en otros campos diferentes, como el de la herencia o el de la presión en líquidos.

Los resultados parecen indicarnos que, tanto en $2^{\circ} \mathrm{co}-$ mo en COU, los alumnos son escasamente consistentes en el uso de la idea de discontinuidad, con coefi. cientes de correlación, que si bien es verdad que llegan a ser significativos $(p<0.01)$ en la mitad de los casos, 
Tabla Ila: Alumnos de $\mathbf{2}^{\circ}$

Consistencia en el tso de la idea de discontinuidad.

\begin{tabular}{|l|cccccc|}
\hline & 1 & 5 & CA & 6 & 9 & 11 \\
\hline 1 & 1 & 0.06 & 0.26 & 0.30 & 0.16 & 0.37 \\
5 & & 1 & 0.25 & 0.19 & 0.28 & 0.27 \\
64 & & & 1 & 0.20 & 0.24 & 0.30 \\
B & & & & 1 & 0.37 & 0.21 \\
7 & & & & & 1 & 0.13 \\
11 & & & & & & 1 \\
\hline
\end{tabular}

Tabla IIb: Alumnos de COU

\begin{tabular}{|c|cccccc|}
\hline 0 & 1 & 5 & 64 & 0 & 9 & 11 \\
\hline 1 & 1 & 0.12 & 0.36 & -0.11 & 0.07 & -0.08 \\
5 & & 1 & 0.35 & 0.09 & 0.03 & -0.09 \\
64 & & & 1 & -0.02 & 0.24 & -0.10 \\
0 & & & & 1 & 0.29 & 0.28 \\
9 & & & & & 1 & 0.17 \\
11 & & & & & & 1 \\
\hline
\end{tabular}

alcanzan valores muy pequeños, inferiores en todos ellos a 0.4. Para perfilar mejor la situación en la que nos estamos moviendo, se ha representado, a modo de ejemplo, las tablas de contingencias correspondientes a las cuestiones 8 y 9 , ya que era éste uno de los casos en el que los alumnos parecian mostrar mayor consistencia en sus respuestas (Tabla III). Como se puede apreciar, solo un escaso porcentaje de los sujetos $37 \%$ en $2^{\circ}$, y $41 \%$ en COU) que usaban la idea de discontinuidad en alguna de las dos cuestiones, to hacían en ambas simultáneamente.

Tabla IH

Tablas de contingencia relativas a la utilización explicita de un modelo corpuscular durante las explicaciones.

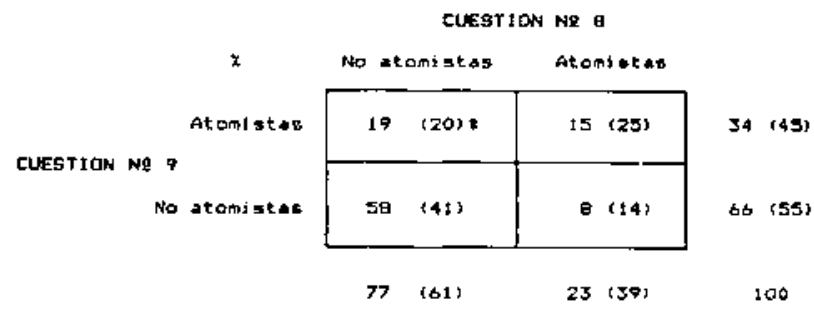

Los datos entre paréntesis corresponden a los alumnos de COU.

\subsection{Nivel de aceptación de la conservación de la masa}

A través de las cuestiones $2,4,9$ y 11 , se trató de determinar hasta qué punto y con qué consistencia los alumnos eran capaces de predecir la conservación de la masa en un fenómeno físico, así como la importancia que los chicos atribuían a la utilización de un modelo corpuscular a la hora de realizar sus predicciones.

Los resultados que aparecen en la Tabla IV nos indican que muy pocos de los alumnos de $2^{\circ}$ utilizaban en

\section{Tabia IV}

Interacción entre el grado de apropiación de la idea de discontinujdad y el nivel de aceptación de la conservación de la masa.

\begin{tabular}{|c|c|c|c|}
\hline CLESTION NQ & $\begin{array}{l}\text { * Al unno* } \\
\text { itamistas }\end{array}$ & $\begin{array}{c}\text { X Al unna: } \\
\text { congervativos }\end{array}$ & 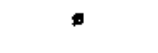 \\
\hline 2 & $15(26)$ & $16\{5\}\}$ & $-0.06(0.10)$ \\
\hline 4 & $15(19)$ & $26159\}$ & $-0.02(0.26)$ \\
\hline 9 & $34(94)$ & 18 (75) & $0.1010,131$ \\
\hline \multirow[t]{2}{*}{11} & $35(7 \bar{\imath})$ & A $(91)$ & $0.13 \quad(0.07)$ \\
\hline & $\overline{25} \overline{4}$ & $24(6)$ & \\
\hline
\end{tabular}

Los datos entre paréntesis corresponden a los alumnos de COU.

sus respuestas la idea de conservación, sobre todo si en el fenómeno al que se hacia referencia se veian in. volucrados gases. En estos casos, se ofrecían argumentos tales como «los gases calientes pesan menos», "los gases pesan menos que los líquidos» 0 «los gases no pesan's. Resultados similares se han obtenido en otros trabajos realizados en España con alumnos valencianos (Furió y Hernández 1983, Furío, Hernández y Harris 1987), a través de los cuales se ha podido constatar que los adolescentes tienen una imagen de los gases como de algo inmaterial, incapaces de intervenir en procesos físicos y químicos.

A pesar de observarse diferencias significativas ( $p<$ 0.01 ) en los porcentajes correspondientes a los alum. nos de COU, tampoco en este caso los resultados son todo lo buenos que sería deseable. Con mucha frecuencia, los estudiantes vuelven a sugerir las mismas argumentaciones erróneas que ofrecian los alumnos de $2^{\circ}$.

Tanto en uno como en otro caso, los estudiantes parecen mostrar, incluso, una escasa consistencia en sus respuestas (Tabla V), de forma que los diversos contextos en los que se mueven cada una de las cuestiones propuestas parecen sugerir a los estudiantes respuestas diferentes. Esto, sin duda, cuestiona la existencia en los sujetos de marcos conceptuales generales con los cuales interpretar fenómenos similares, lo que parece sugerir la idea de que los alumnos interpretan los fenomenos cotidianos al margen de las teorias, modelos y principios que se estudian en la escuela. 
Tabla $\mathrm{V}$

Consistencia en el uso de la idea de consrvación.

\begin{tabular}{|c|cccc|}
\hline & 2 & 1 & 0 & 11 \\
\hline 2 & 1 & 0.14 & 0.18 & 0.12 \\
& & 10.3018 & 10.41 & $1-0.001$ \\
4 & & 1 & 0.33 & 0.10 \\
9 & & & $(0.10)$ & $1-0.08)$ \\
12 & & & 1 & 0.07 \\
& & & & $0.03)$ \\
\hline
\end{tabular}

Los datos entre parentesis corresponden a los alumnos de COU

Igualmente, la Tabla IV muestra de forma comparativa los porcentajes de alumnos atomistas junto con los porcentajes de alumnos conservativos, así como los correspondientes coefiencies de correlación $(\varnothing)$, obtenidos cn cada caso. Como puede apreciarse, a pesar de que la enseñanza recibida a to largo del bachillerato contribuye a aumentar de forma sustancial, aunque no espectacularmente, el nivel de aceptación de la conservación, aporta muy poco a la adquisición de un modelo explicativo como es el modelo corpuscular de la materia. Los bajos coeficientes de correlación inter-ítems obteridos en estos casos no hacen sino apoyar esta idea, ya que a nivel global, tanto en $2^{\circ}$ como en COU, los sujetos parecen encontrar una escasa relación entre el tipo de respuesta que dan en cada pregunta y el modelo que utilizan durante la explicación.

A modo de ejemplo, para ilustrar este aspecto, se han recopilado en la Tabla VI algunas de las explicaciones más representativas dadas por los alumnos a to largo de la cuestión 9. Como puede observarse, los estudiantes se pronuncian o no a favor de la conservación de la masa independientemente de si utilizan o no un modelo microscópico en sus razonamientos.

Tabla VI: Alumnos de $2^{\circ}$ de BUP y de COU

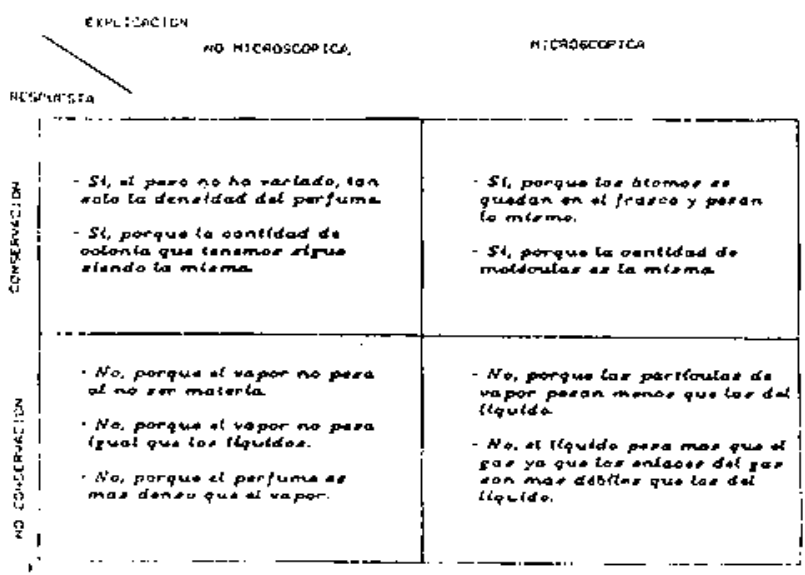

\section{CONCLUSIONES}

Tal como se ha tratado de mostrar, los alumnos que comienzan la Química en el bachillerato parecen tener escasamente asumida la discontinuidad de la materia, lo que se traduce en un reducido número de explicaciones sustentadas sobre un modelo corpuscular, así como un escaso grado de consistencia en las respuestas. Así, por ejemplo, muchos de los alumnos que parecen comportarse como atomistas en unas situaciones dadas, parecen no hacerlo en otras distintas.

Por otro lado, la enseñanza recibida a través de los cursos de $2^{\circ}, 3^{\circ}$ y COU parece contribuir muy escasamente a mejorar esta situación, observándose solo en algunos casos un aumento sustancial en la tendencia a utilizar un modelo microscópico durante las explicaciones. Todo esto parece sugerir que gran parte de los esfuerzos del profesorado que imparte Química durante el bachillerato debería centrarse en tratar de propiciar la construcción, por parte de los alumnos, de una auténtica representación discontinua de la materia, ya que gran parte de las dificultades con las que se enfrentan los alumnos en Química durante el bachillerato pudiera derivar de una escaso grado de comprensión, a niveí microscópico, de los fenómenos estudiados así como de las representaciones simbólicas utilizadas.

Tal proceso de asimilación se ve dificultado, si como denuncian algunos autores (Caamaño et al. 1983) se produce una aceptación de partida acrítica del carácter discontinuo de la materia, lo cual parece suceder en la práctica mucho más de lo que sería deseable. Por tanto, la introducción de estos conceptos debiera ir acompanada de un enfoque experimental que aportase a los alumnos una base empírica de apoyo para la construcción de una imagen discontinua de la materia. En este sentido, queremos salir al paso de aquellos enfoques que conciben el aprendizaje como un redescubrimiento autónomo por parte del alumno, mostrándonos más bien partidarios de aquellos enfoques que conciben el aprendizaje como un redescubrimiento orientado hacia un cambio conceptual y metodológico (Gil y Carrascosa 1985).

Por último, diremos que no hemos apreciado interacción alguna entre el uso de un modelo corpuscular y el nivel de aceptación de la conservación de la masa en un fenómeno físico. Esto se puede interpretar teniendo en cuenta el excesivo carácter operativo y mecánico que se suele atribuir a los principios, modelos y teorías científicas que se estudian en la escuela y que, prácticamente, solo son dotados de un valor cuantita" tivo a través de la resolución de problemas numéricos. Se desprende de esto, pues, que los cursos de bachillerato deberían contribuir más a dar una adecuada ima. gen cualitativa de la materia, huyendo del operativismo ciego de algunos tipos de cálculos numéricos en el que se suele incurrir con una cierta frecuencia en las clases de Química. 


\section{REFERENCIAS BIBLIOGRÁFICAS}

ANDERSON, B., 1984. Chemical reactions. EKNA-report $\mathrm{N}^{\circ} 12$ (Departament of Education and Educational Research. University of Goteborg).

BROOK, A., BRIGGS, H, y DRIVER, R., 1984. Aspects of secondary students' understanding of the particulate nature of matter. Children's Learning in Science Project. (Centre for Studies in Science and Mathematics Education. University of Leeds).

CAAMAÑo, A., MAYOS, C., MAESTRE, G. y VENTURA, T., 1983. Consideraciones sobre algunos errores conceptuales en el aprendizaje de la Qujmica en el bachillerato. Enseñanza de las Ciencias, Vol. l(3), pp. 198-201.

DRIVER, R., 1986. Psicología cognoscitiva y esquemas conceptuales de los alumnos. Enseñanza de las Ciencias, Vol. 4(1), pp. 3-15.

ENCISO, E., LLORENS, J.A. y SENDRA, F., 1987. La introducción al modelo corpuscular de la materia. Un estudio evolutivo. Ensehanza de las Ciencias, Vol. extra, pp. 183-184.

ENGEL CLOUGH, E. y DRIVER, R., 1986. A study of consistency in the use of students' conceptual frameworks across different task contexts. Science Education, Vol. 70 (4), pp. 473-496.

FURIO, C. y HERNANDEZ, J., 1983. Ideas de los adolescentes de 11-15 aflos acerca de los gases. Enseñanza de las Ciencias, Vol. l(1), pp. 83-91.

FURIÓ, C., HERNÁNDEZ, J. y HARRIS, H., 1987. Parallels between adolescents' conception of gases and history of chemistry. Journal of Chemical Education, Vol. 64(7), pp. 616-618.
GARRET, H.E., 1983. Estadistica en psicología y educación (Paidos: Barcelona).

GIL, D. y CARRASCOSA, J., 1985. Science learning as a conceptual change and methodological change. European Journal of Science Education, Vol. 7(3), pp. 231-236.

LLORENS, J.A., 1987, La concepción corpuscular de la materia en los alumnos que comienzan el estudio de la quimica en las ensexananzas medias, en Álvarez A. (comp.), Psicología y educación. Realización y tendencias actuales en la investigación y en la práctica. (MEC y Visor: Madrid).

MARTINAND, J.L., 1986. Ensenanza y aprendizaje de la modelización. Enseñanza de las Ciencias, Vol. 4(1), pp. 45-50.

MITCHELL, A.C. y KELLINGTON, S.H., 1982. Learning difficulties associated with the particulate theory of matter in Scottish Integreted Science course. European Journal of Science Education, Vol, 4, pp. 429-440.

NOVICK, S. y NUSSBAUM, J., 1978. Junior high school pupil's understanding of the particulate nature of matter: An interview study. Science Education, Vol, 62(3), pp. 273-282.

NOVICK, S. y NUSSBAUM, J., 1981. Pupil's understanding of the particulate nature of matter: A cross-age study. Science Education, 65(2), pp. 187-196.

ROSADO, L., 1986. Modelos en los procesos de la ciencia y su constrastación. (UNED: Madrid).

SAMPASCUAL, G., 1982. Las pruebas objetivas. (Anaya: Madrid). 


\section{ANEXO}

10) Tenemos dog recipientes 1gualas, uno lleno de ague lfquida y otro de vapor de agua. Mediante une balanze observamos que el reokpiente que contione gue liquide es más pesedo que el que contiene vapor. ¿Cóno es posible que dos volimenes de la misma sustancla tengrn distinto peso?.

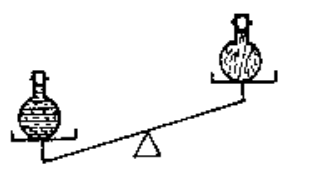

29) Colocamos a Embos lados de una balanza dos recipientes 11 enos de alre hembticamente cerrom dos. In1cialmente la balianza oncuentra equilibmada, S1 modiante una vela calentawoo ol recipiente de la derecha, ise dentvolara la balane 8 ?.

s1.... [

No.....

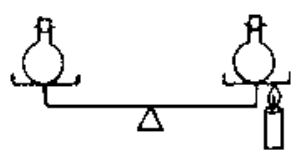

Explice la contestación que heg dedo.

39) Bh recipiente de la 1zquierda esta lleno de van sas rojo (díxido de carbono) que ropreanter remos medtante

In de la dereche, egtá llano de un gas incoloro (aire) que representaremos mediento 曷福

-Mbuja qué es lo que queda en log depósitos degpués de

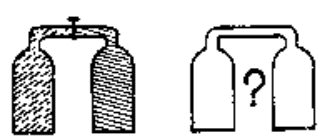
un rato de haber abjerto 1 a liave.

- Explica cómo y por qué tiene lugar el fenbmeno.

42) Imagine una jer-1nga de pĺást1co con al go de atre en su interlor y el orfficio de alida teper do'

-Dibuja la posición aproxiarada de 1 finpolo de la jerinGa tra ver que éfta se Introduoe on un balo de agra oelionta.

- Llis cambiado ol peso del nil re que contenía la farlnge? -Explicar la rapuesta.

58) En ol deposito de 1 a figura tenemos alro confinado mediante un émbolo sobre el que colocamo pesas para corpensar la presión interior que hay en el reclolente. Nediante una llama calentamos al dep6e1to observando que, a medida que transourre el, t1empo, debemos colocar sobre el ́́mbolo un mayor nímero de pesas a1 queremos mantenoxio siempre en la misma posiaion.
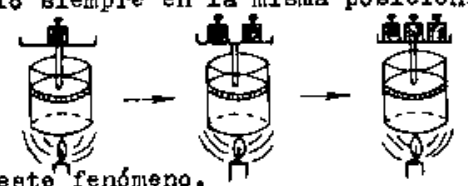

-Explce esto fenoneno.

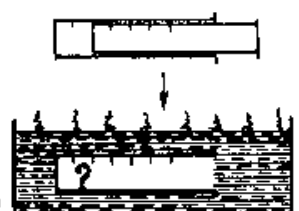

69) Ia figura adfunte representa una jeringa 11ena da alre cujo orificto de salida ha sido obetruido. Si anadimos Feses sobro ol Embolo el aire se oocprime.

a) Explica a que ge dabe le fact-

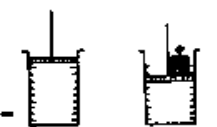
Liand can que se comprime el aire.

b) 2 guo diferencias observariemos 81 la jeringa e tuviege llena de Bgue en lugar de atre?. Bxplica la respuesta. 7a) ¿Crees que serla posible obtener agua 1fqu1da, en un recipiente cerrado, comprimfendo un poco de vapor de agua?.

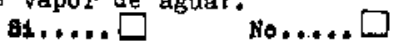
In oaso artrmativo explicar corno es posible que ocurra aloo asf. En caso negativo, explicar por qué.

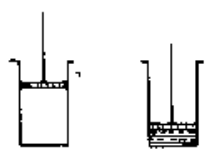

89) Cuando destapas un erasco de perfume al cabo do cierto tiempo se nota au aroms. Si el perfume eatá en el tarrito y ea un

I quido, zcóno se percibe au preasncia en toda la habitac18n?.

¿Cómo explicas que el perfume Ifquido ooupe un volumen pequefio mientres au arome parece llenar toda la habltac16n?.

98) Tonemos un fragquito cerrado con un poco do perfume en au interior. Colocaros el frasquito en uno de $10 a$ platilios do la balanza que se equilibra volocando pesaa en el atro platillo.

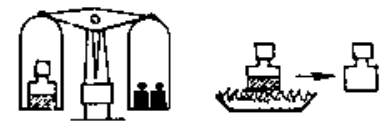

Celentamos luego el frasco cemado y el perfume pase a vator. Al volver a colocarlo sobre la balanza, ¿Permaneceré Gsta equilibrada?.

-Explicacion.

S1..... No.... $\square$

-Dibuja en el gráf100 adjunto como se distrtbuye en el tarro el vapor obtenido al

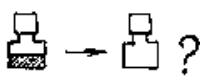
calentar el porfume?.

-Explicación.

109) Ouando termina el Invierno las mujeres suelen colocar on 108 roperos, trajes y abrigos unas pestillas de una suetancia edilds (naftalina) para preservarle cortra le polilla.

- Bxplicar los aiguientes fenómenos que pueden observarae al reapecto:

a) En olor que omiten diohos solidos.

b) Al cabo do clerto t1empo se puede comprobar que las pastillas se ha reducido y cada vez pesan menos.

128) Sobre uno de log platillos de una balanza colocamos un poco de sal $\mathrm{y}$ un reciplente con agua. A continuación equilibramos le balanza colocando pesas en el otro platilio. Echamos la sal sobre el agua $y$ a ls vez agitamos, observando poco después que el agua tiene sabor salado $y$ que sin embargo la sal no se ve.

- ¿ Seguirá la balanza equi-

librada al final de la ope-

rac16n que hemos realiado?

s1......

-Explica detalladamente qué

piensas que le he ocurrido

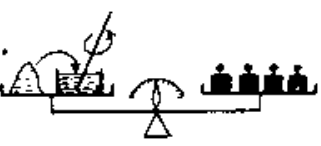

a 1 s gal.

-\$1 la solución obtenida la

fil tremos, ¿seguirá tentendo

sabor saledo ol liquido que

se recoge?

si......

No.....

122) ¿ckmo explicarias que liquidos distinto como el agua y el alcohol tengan diatinta capac1dad pare eveporares? 